\title{
Caracterisation des performances de croissance et de resistances aux maladies et aux ravageurs de sept (7) varietes \\ de patate douce (Ipomoea Batatas (l), Lam., 1793) dans la region de bouake, Cote d'Ivoire
}

\author{
Kouame N'guessan \\ Université Jean Lorougnon GUEDE (UJLoG), Laboratoire d'Amélioration \\ de la Production Agricole (LAPA), UFR Agroforesterie, Côte d'Ivoire \\ Dibi Konan Evrard Brice \\ Essis Brice Sidoine \\ Centre National de Recherche Agronomique (CNRA), Programme Plantes à \\ Racines et Tubercules (PRT), Station de Recherche sur les Cultures \\ Vivrières (SRCV), Côte d'Ivoire \\ Toure Nin Céline \\ Université Jean Lorougnon GUEDE (UJLoG), Laboratoire d'Amélioration \\ de la Production Agricole (LAPA), UFR Agroforesterie, Côte d'Ivoire \\ Ngbesso Mako François De Paul \\ Centre National de Recherche Agronomique (CNRA), Programme des \\ Cultures Maraichères (PCM), Station de Recherche sur les Cultures \\ Vivrières (SRCV), Côte d'Ivoire
}

Doi:10.19044/esj.2021.v17n10p51

Submitted: 30 September 2020

Accepted: 23 March 2021

Published: 31 March 2021
Copyright 2021 Author(s)

Under Creative Commons BY-NC-ND

4.0 OPEN ACCESS

Cite As:

N'guessan K., Evrard Brice D.K., Brice Sidoine E., Nin Céline T. \& François De Paul N.M. (2021). Caracterisation des performances de croissance et de resistances aux maladies et aux ravageurs de sept (7) varietes de patate douce (Ipomoea Batatas (l), Lam., 1793) dans la region de bouake, Cote d'Ivoire. European Scientific Journal, ESJ, 17(10), 51.

https://doi.org/10.19044/esj.2021.v17n10p51

\section{Résumé}

La production de la patate douce, Ipomoea batatas (L.) Lam., reste confrontée à de nombreuses contraintes agronomiques. Pour mieux faire face à ces contraintes, une étude a été réalisée dans le but de déterminer les caractères agronomiques de sept (7) variétés de patate douce cultivée au centre de la Côte d'Ivoire (Bouaké) afin de sélectionner les variétés les plus adaptées. 
Dans cette étude, le taux de survie, la biomasse aérienne, la résistance aux insectes et ravageurs ont été évalués. Les résultats obtenus ont montré que les variétés Covington, CIP et Irène ont enregistré les meilleurs taux de survie. Au niveau de la biomasse aérienne la variété Irène s'est distinguée des autres variétés. La variété Fadanga a été moins sensibles pour l'ensemble des nuisibles influençant la patate douce.

Mots-clés : Patate douce, variétés, caractères agronomiques, sélection, performance

\title{
Characterization of Growth Performance and Resistance to Diseases and Pests of Seven (7) Varieties of Sweet Potato (Ipomoea Batatas (L), Lam., 1793) in the Bouake Region, Cote d'Ivoire
}

\section{Kouame N'guessan}

Université Jean Lorougnon GUEDE (UJLoG), Laboratoire d'Amélioration de la Production Agricole (LAPA), UFR Agroforesterie, Côte d'Ivoire

\section{Dibi Konan Evrard Brice}

\section{Essis Brice Sidoine}

Centre National de Recherche Agronomique (CNRA), Programme Plantes à Racines et Tubercules (PRT), Station de Recherche sur les Cultures Vivrières (SRCV), Côte d'Ivoire

\section{Toure Nin Céline}

Université Jean Lorougnon GUEDE (UJLoG), Laboratoire d'Amélioration de la Production Agricole (LAPA), UFR Agroforesterie, Côte d'Ivoire

\section{Ngbesso Mako François De Paul}

Centre National de Recherche Agronomique (CNRA), Programme des Cultures Maraichères (PCM), Station de Recherche sur les Cultures

Vivrières (SRCV), Côte d'Ivoire

\begin{abstract}
The production of the sweet potato, Ipomoea batatas (L.) Lam., still faces many agronomic constraints. To better face these constraints, a study was carried out in order to determine the agronomic characteristics of seven (7) varieties of sweet potato cultivated in the center of Côte d'Ivoire (Bouaké) in order to select the most suitable varieties. In this study, survival rate, aboveground biomass, resistance to insects and pests were assessed. The results obtained show that the Covington, CIP, and Irene varieties recorded the best survival rates. In terms of aerial biomass, the Irene variety stood out
\end{abstract}


from other varieties. The Fadanga variety was less susceptible to all the pests influencing the sweet potato.

Keywords: Sweet potato, varieties, agronomic character, selection, performance

\section{Introduction}

La patate douce, Ipomea batatas est une plante dicotylée appartenant à la famille des convolvulaceae. Cette famille comporte plus ou moins 50 genres et plus de 1000 espèces. Parmi ces espèces, seule Ipomea batatas revêt une importance économique et elle est aussi consommée comme salade ou légume vert (Amani, 2017). Sa culture se fait dans les régions tropicales et subtropicales telles que l'Amérique Centrale (le Pérou ou les Philippines). Ipomea batatas comprend des milliers de variétés qui se distinguent par le port et la couleur des tiges, la forme, le type, la taille et la couleur des pétioles et des feuilles, ainsi que par la peau et la chair des racines tubéreuses, etc.

La patate douce constitue la septième ( $\left.7^{\text {ième }}\right)$ culture alimentaire dans le monde après le blé, le riz, le maïs, la pomme de terre, l'orge et le manioc (Hironori et al., 2007). Elle est la seconde plante à racines et à tubercules après le manioc dans les pays tropicaux (FAOSTAT, 2006) et la troisième ( $3^{\text {ième }}$ ) en Afrique subsaharienne après le manioc et l'igname (Karyeija et al., 1998). L'Asie enregistre la plus grande production avec plus de $75 \%$, puis l'Afrique avec $20 \%$, l'Amérique $4 \%$ et l'Europe inférieur à $1 \%$. Les superficies emblavées sont de 4,02 millions d'hectares en Asie et de 3,74 millions d'hectares en Afrique. Les rendements moyens varient de 5,4 t/ha (Afrique) à 19,5 t/ha (Asie). En 2011, environ 8 millions d'hectares de terres cultivables dans le monde ont été utilisées pour produire la patate douce dont plus de 95 $\%$ de la production mondiale provenait des pays en voie de développement. La Côte d'Ivoire avait une production de 47914 tonnes en 2013 (FAOSTAT, 2018). Cette production est essentiellement composée de patate douce à chair blanche et jaune. La patate douce est cultivée dans toutes les régions du pays. Elle est consommée sous diverses formes (légume, frite, bouillie, foutou, etc.).

Cependant, de nombreux facteurs limitent la production de cette culture. Ce sont entre autres : la perte progressive des variétés traditionnelles, la baisse de la fertilité des sols, le manque de variétés à haut potentiel de rendement, la pression parasitaire, le manque de disponibilité de bonnes semences en temps utile et le faible niveau d'adoption des technologies mises au point par la recherche. Pour résoudre ces problèmes, il est initié par le Centre National de Recherche Agronomique (CNRA) en collaboration avec Helen Keller International (HKI) un programme de sélection des variétés performantes. La sélection de ces variétés est faite selon leur vigueur, leur résistance aux maladies et ravageurs, leur teneur en vitamine A, leur 
rendement, leur état sanitaire, leur aptitude à la cuisson et leur goût. C'est ce qui justifie notre travail dont l'objectif est de déterminer les performances de croissance et de résistances aux maladies et aux ravageurs des différentes variétés de patate douce.

\section{Matériel Et Méthodes}

\subsection{Zone D'étude}

Bouaké, appelée Gbékékro jusqu'en 1900, est une ville du centre de la Côte d'Ivoire, située à $350 \mathrm{~km}$ environ d'Abidjan (Yesso et al., 1991). La ville est sur un relief plat, avec une importante constellation de 143 villages dénombrés dans un rayon de 20 kilomètres. La végétation de la région est recouverte d'une savane boisée et traversée par la rivière Kan. Cette ville est située à la latitude $7^{\circ} 69 \mathrm{~N}$ et longitude $5^{\circ} 03 \mathrm{O}$, s'étend sur une superficie d'environ $72 \mathrm{~km}^{2}$ (Allouko, 2011). Elle est influencée par un climat tropical humide. En plus d'une amplitude thermique élevée $\left(22^{\circ} \mathrm{C}\right.$ les matins à $35^{\circ} \mathrm{C}$ les après-midi), d'un ensoleillement constant et d'une hygrométrie faible, on distingue aussi quatre saisons pour la ville de Bouaké (Yesso et al., 1991). On a une saison chaude, sèche et non pluvieuse (novembre à février), une saison chaude, humide et pluvieuse (mars à juin), une saison fraîche, humide et peu pluvieuse (juillet à août) et enfin une saison fraîche, humide et pluvieuse (septembre et octobre).

\subsection{Matériel végétal}

Le matériel végétal utilisé était constitué de sept (7) variétés de patate douce que sont : Fadanga ; Pourpre (T9-Purple) ; Covington ; Tiébélé 2 ; CIP (CIP19906261) ; CIP4 (BF52XCIP4) et la variété Irène.

\subsection{Dispositif expérimental}

Le dispositif expérimental (figure 1) est un bloc de Fisher comportant un seul facteur à trois répétitions. La parcelle élémentaire d'une longueur de 5 $\mathrm{m}$ et d'une largeur de $3 \mathrm{~m}$ est composée de 24 buttes. La distance entre deux parcelles élémentaires est de $1,5 \mathrm{~m}$. La distance entre deux répétitions consécutives est de $2 \mathrm{~m}$. Ainsi une répétition comporte sept parcelles élémentaires représentant les sept variétés. La répétition a été réalisée sur une longueur de $44 \mathrm{~m}$ et une largeur de $3 \mathrm{~m}$. L'expérimentation est faite sur une superficie de $572 \mathrm{~m}^{2}$. Pour cette expérimentation, aucun fertilisant n'a été utilisé. 


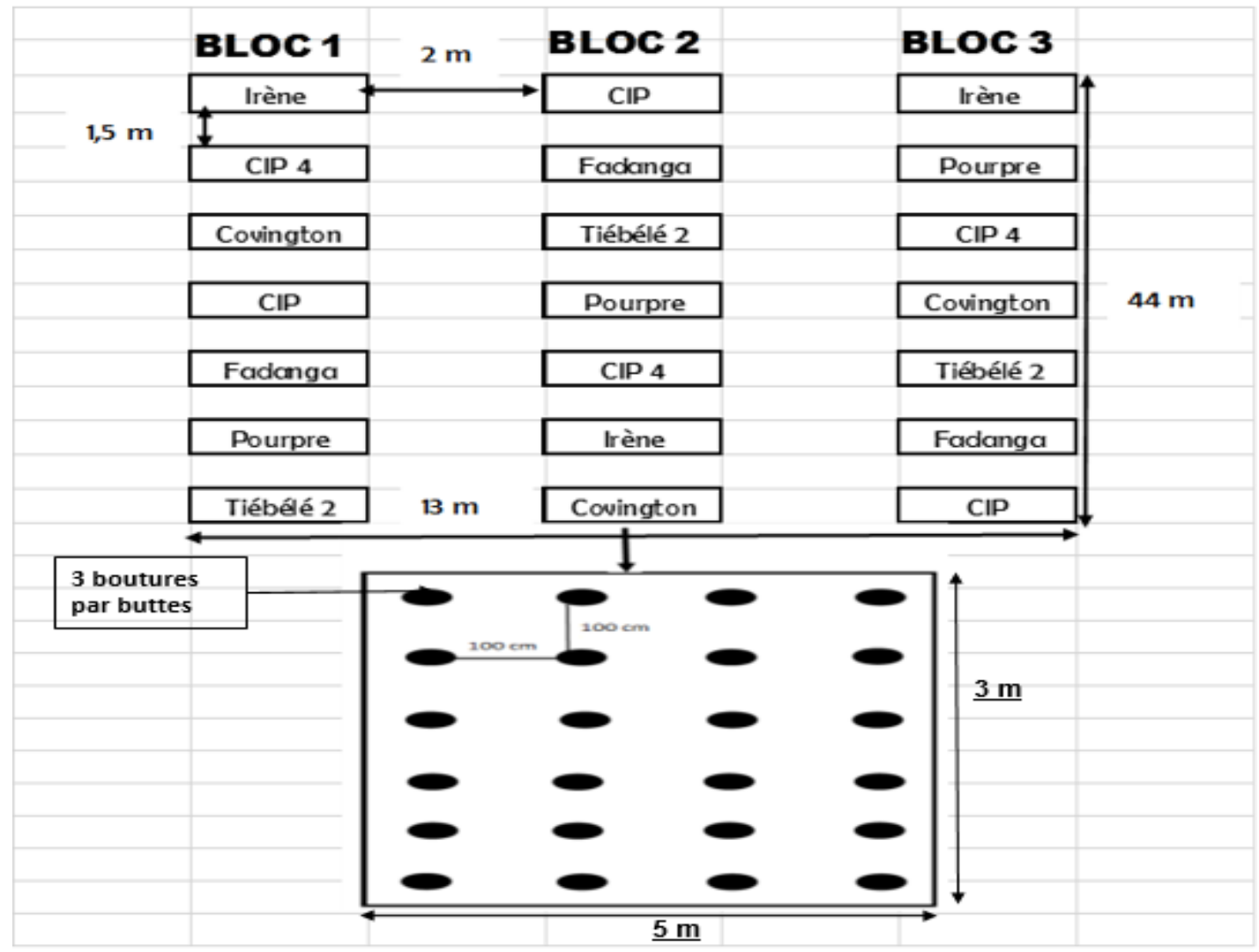

Figure 1. Dispositif expérimental

\subsection{Mise en place des cultures}

Le site a préalablement été désherbé. Ensuite, le piquetage et le labour ont été effectués avant la confection des buttes de $1 \mathrm{~m}^{2}$. Des boutures de quatre (4) nœuds ont été coupées puis plantées sur des buttes. Ces boutures d'une longueur d'environ $30 \mathrm{~cm}$ ont été effeuillées avant d'être repiquées à raison de trois boutures par butte. Les boutures ont été repiquées de façon oblique avec 2 nœuds dans le sol et 2 nœuds hors du sol. Le repiquage et la coupure des boutures ont été effectués le même jour. Ce repiquage a été fait variété par variété et bloc après bloc.

\subsection{Entretien du champ}

L'entretien de la parcelle a été fait par sarclage manuel, régulièrement réalisé afin de débarrasser le champ des mauvaises herbes.

\subsection{Collecte des données}

Plusieurs paramètres ont été observés pendant le déroulement de l'essai. Il s'agit du taux de levé des plants, du poids de la biomasse aérienne 
fraiche, de l'incidence et de la sévérité des virus et enfin de l'incidence des attaques des ravageurs et des charançons sur les variétés de patate douce.

\section{Taux de reprise (Tr)}

Après le repiquage des plants, l'émission de nouvelles feuilles et la survie des boutures traduisent la reprise de leur croissance. Ainsi un mois après le repiquage des boutures, le nombre de plant levé a été compté afin de déterminer le taux de reprise selon la formule suivante :

$$
\mathrm{T} r=\frac{\text { Nombre de Plant Levé (NPL) }}{\text { Nombre de Plant repiqués (NPPE) }} * 100
$$

\section{Le poids de la biomasse aérienne (PBA)}

Le poids de la biomasse aérienne a été mesuré à la récolte en sectionnant les tiges à l'aide d'une paire de ciseaux suivi de leurs pesées ;

\section{Incidence des viroses (IxPV)}

Deux observations ont été faites pour voir l'état sanitaire des plants. La première a eu lieu 6 semaines après la plantation et la deuxième, 3 mois après la plantation. Ainsi le taux de plants virosés (TxPV) se traduisant par le nombre de plants virosés (NPV) sur le nombre total de plant (NTP) est déterminé par la formule suivante :

$$
\mathrm{IxPV}=(\mathrm{NPV} / \mathrm{NTP}) \mathrm{x} 100
$$

\section{Indice de sévérité des viroses}

La sévérité de la maladie a également été déterminé à l'aide d'une feuille de notation allant de 1 à 9 établit pour la patate douce :

1. pas de symptômes viraux ;

2. symptômes viraux peu clairs ;

3. symptômes viraux clairs $5 \mathrm{pc}$ des plantes par parcelle ;

4. symptômes viraux clairs chez $6-15 \mathrm{pc}$ des plantes par parcelle ;

5. symptômes viraux clairs chez $16-33$ pc des plantes par parcelle;

6. symptômes viraux clairs chez 34- 66 pc des plantes par parcelle (plus de $1 / 3$; moins de $2 / 3$ );

7. symptômes viraux clairs chez $67-99$ pc des plantes par parcelle $(2 / 3$ à presque toutes) ;

8. symptômes viraux clairs chez toutes les plantes par parcelle (non rabougris);

9. symptômes viraux sévères chez toutes les plantes par parcelle (retard de croissance). 


\section{Incidence des ravageurs (InRav)}

Il a été calculé en faisant le quotient du nombre de racines tubéreuses attaqués par les ravageurs (tubercules comprenant des galeries faites par les rongeurs) sur le nombre total de racines tubéreuses ; le tout multiplié par cent $(100)$;

$$
\text { InRav }=\frac{\text { Nombre de racines attaqués par les ravageurs }}{\text { Nombre total de racines }} * 100
$$

\section{Incidence des attaques de charançons (InCharn)}

Il a été déterminé en faisant le quotient du nombre de racines tubéreuses attaqués (tubercules comprenant des galeries faites par les larves de charançons) sur le nombre total de racines tubéreuses ; le tout multiplié par cent (100) ;

$$
\text { InCharn }=\frac{\text { Nombre de racines attaqués par les charançons }}{\text { Nombre total de racines }} * 100
$$

\subsection{Traitement de données}

Les données collectées ont fait l'objet d'analyse de variance (ANOVA) à un facteur avec sept (7) variables à l'aide du logiciel Statistica 7.1. Cette analyse a été utilisée pour évaluer l'effet de la variété sur les paramètres mesurés de la patate douce. En cas de différences significatives au seuil de $5 \%$, le test de Fisher a été utilisé pour classer les moyennes en groupes homogènes.

\section{Résultats}

\subsection{Taux de survie}

Les taux de survie des plants de chaque variété aux différents stades de croissance de la patate douce (repiquage jusqu'à la récolte) sont présentés par le Tableau 1. L'analyse de ce paramètre aux différents stades de développement de la patate douce a révélé une différence significative entre les variétés $(\mathrm{P}<0,001)$.

Au stade plantule, le taux de survie le plus faible $(20,83 \pm 7,73 \%)$ est enregistré avec la variété Fadanga pendant que la plus grande valeur de ce paramètre $(90,74 \pm 3,49 \%)$ est obtenue avec la variété Irène. A ce stade, le taux de survie des variétés CIP 4, Pourpre et Tiébélé 2 présentent une différence significative à celui de la variété Irène. Le taux de survie entre les variétés Irène, Covington et CIP n'est pas significativement différent.

Au stade floraison, les taux de survie varient de 14,81 $\pm 4,46 \%$ (variété Fadanga) à 79,17 $\pm 6,36 \%$ (variété CIP). Le taux de survie n'est pas 
significativement différent entre les variétés Irène, Covington et CIP. Cependant, les taux de survie des variétés CIP 4, Fadanga, Tiébélé 2 et Pourpre sont significativement différents de celui de la variété Irène.

A la production, les taux de survie varient de 7,4 $\pm 1,6 \%$ (variété Fadanga) à 50,92 $\pm 18,07 \%$ (variété Covington). A ce stade, d'une part, les taux de survie des variétés Irène et CIP ne présentent pas de différence significative de même que ceux des variétés CIP 4 et Pourpre d'autre part. Par ailleurs, les taux de survie des variétés CIP4, Fadanga, Tiébélé et Pourpre sont significativement différents de celui de la variété Irène. Cependant, les taux de survie des variétés Fadanga et Tiébélé ne sont pas significativement différents. Dans l'ensemble, du stade plantule à la production le taux de survie de la variété (Irène) a baissé d'au moins $20 \%$ en passant d'un stade à un autre.

Tableau 1. Taux de survie des plants des différentes variétés selon les stades de croissance de la patate douce

\begin{tabular}{|c|c|c|c|c|}
\hline Variétés & NPPE & Stade plantule (\%) & Stade de floraison $(\%)$ & Production (\%) \\
\hline IRENE & 72 & $90,74 \pm 3,49^{a}$ & $69,44 \pm 11,11^{\mathrm{a}}$ & $40,27 \pm 7,35^{\mathrm{a}, \mathrm{b}}$ \\
\hline CIP 4 & 72 & $43,12 \pm 2,87^{b}$ & $32,4 \pm 3,49^{\mathrm{b}}$ & $19,44 \pm 6,05^{\mathrm{c}}$ \\
\hline COVINGTON & 72 & $78,24 \pm 8,92^{\mathrm{a}}$ & $65,27 \pm 8,33^{\mathrm{a}}$ & $50,92 \pm 18,07^{\mathrm{a}}$ \\
\hline CIP & 72 & $89,35 \pm 5,61^{\mathrm{a}}$ & $79,17 \pm 6,36^{\mathrm{a}}$ & $41,2 \pm 3,49^{\mathrm{a}, \mathrm{b}}$ \\
\hline FADANGA & 72 & $20,83 \pm 7,73^{\mathrm{c}}$ & $14,81 \pm 4,46^{\mathrm{c}}$ & $7,4 \pm 1,6^{\mathrm{d}}$ \\
\hline POURPRE & 72 & $50,15 \pm 9,88^{b}$ & $33,8 \pm 13,70^{\mathrm{b}}$ & $20,37 \pm 2,12^{c}$ \\
\hline TIEBELE 2 & 72 & $52,31 \pm 8,37^{\mathrm{b}}$ & $37,03 \pm 2,12^{\mathrm{b}}$ & $13,42 \pm 3,21^{\mathrm{d}}$ \\
\hline$P(A N O V A)$ & & 0,000001 & 0,000001 & 0,000087 \\
\hline
\end{tabular}

Les moyennes portant les mêmes lettres ne présentent pas de différences significatives selon les tests d'ANOVA et LSD de Fisher au seuil de $5 \%$. NPPE : Nombre de Plants Repiqués.

\subsection{Biomasse aérienne des variétés de la patate douce}

La figure 2 présente la biomasse aérienne de chaque variété. La biomasse aérienne varie d'une variété à une autre. La variété Irène a produit la plus forte biomasse aérienne par rapport aux six (6) autres variétés. Sa biomasse aérienne est d'environ (10 kg/ha). La plus faible biomasse aérienne a été obtenue par la variété Tiébélé $2(2,33 \mathrm{~kg} / \mathrm{ha})$. De même, les variétés Fadanga $(3,5 \mathrm{~kg} / \mathrm{ha})$ et CIP $4(3,33 \mathrm{~kg} / \mathrm{ha})$ ont également des biomasses aériennes faibles comparativement à la variété Irène. Par ailleurs, les variétés CIP, Covington et Pourpre ont donné des biomasses aériennes moyennes respectivement de $5,56 \mathrm{~kg} / \mathrm{ha}, 6,66 \mathrm{~kg} / \mathrm{ha}$ et $6,18 \mathrm{~kg} / \mathrm{ha}$. 


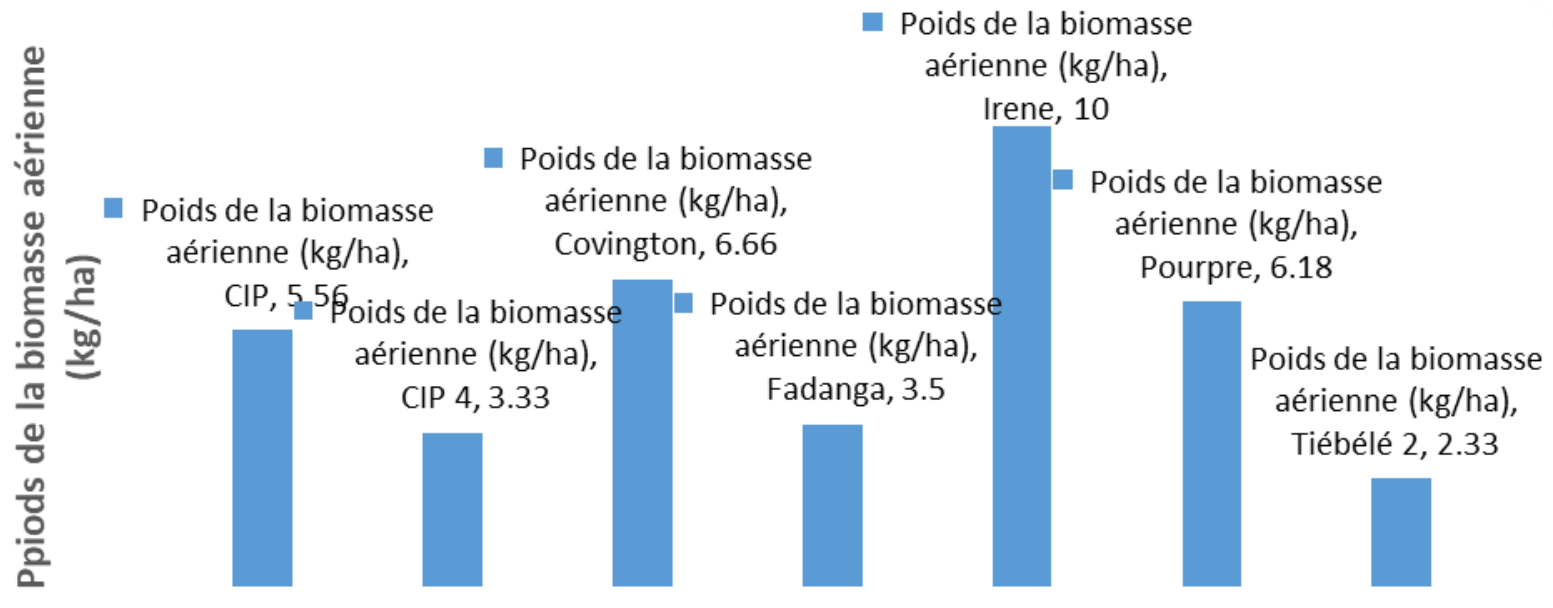

\section{Variétés}

Figure 2. Biomasse aérienne en fonction des variétés de patate douce

\subsection{Incidence des ravageurs, des charançons et des virus sur les $v$ ariétés de patate douce}

Le Tableau 2 présente les différentes attaques de maladie et ravageurs subies par chaque variété. Une différence hautement significative a été enregistrée entre les variétés concernant les attaques induites par les charançons (insectes) $(\mathrm{P}<0,001)$. Les valeurs moyennes des attaques par ces derniers montrent que la variété Fadanga est la moins attaquée $(5 \pm 3,60)$ alors que la variété Covington est la plus attaquée $(45,33 \pm 11,37)$. Les effets des attaques des charançons sur les variétés Irène $(11,67 \pm 15,31)$, CIP 4 (11 \pm 9,64), Fadanga $(5 \pm 3,60)$ et Tiébélé $2(6 \pm 4,58)$ ne sont pas significativement différents $(\mathrm{P}>0,05)$. Hormis les variétés Covington et CIP, les autres variétés ont pratiquement eu la même sensibilité que Irène face aux charançons.

Quant à l'influence des autres ravageurs, des différences significatives ont été observées au niveau des différentes variétés. Les variétés CIP 4, Fadanga, Pourpre et Tiébélé 2 n'ont subi aucune attaque de ces derniers. La variété Covington (01 tubercule attaqué) a été la plus attaquée. Les variétés CIP et Irène ont connu les mêmes dégâts face aux ravageurs. Les moyennes d'attaque sont à environ $0,67 \pm 0,58$ chez ces deux dernières.

Une différence significative a été observée entre les variétés au niveau de la sévérité des viroses $(\mathrm{P}<0,001)$. Elle a varié de $2 \pm 0$ (variétés CIP 4 et Fadanga) à 7,66 $\pm 0,58$ (variété Tiébélé 2). Les variétés CIP 4, Covington et Fadanga ne présentent pas de différence significative. Ces variétés ont été moins virosés par rapport aux autres variétés. L'effet des virus a été plus sévère chez les variétés Tiébélé 2, CIP et Pourpre que chez Irène, 
contrairement aux variétés Covington ; Fadanga et CIP4 chez lesquelles celuici a été moins sévère.

Tableau 2. Influence des charançons, ravageurs et des virus sur les variétés

\begin{tabular}{|c|c|c|c|}
\hline \multirow[b]{2}{*}{ Variétés } & \multirow[b]{2}{*}{ Charançons (\%) } & \multirow[b]{2}{*}{ Ravageurs (\%) } & Virus \\
\hline & & & Sévérité \\
\hline IRENE & $11,67 \pm 15,31^{\mathrm{c}}$ & $0,67 \pm 0,58^{\mathrm{a}, \mathrm{b}}$ & $4 \pm 0^{c}$ \\
\hline CIP 4 & $11 \pm 9,64^{c}$ & $0 \pm 0^{\mathrm{b}}$ & $2 \pm 0^{\mathrm{d}}$ \\
\hline COVINGTON & $45,33 \pm 11,37^{\mathrm{a}}$ & $1 \pm 0^{\mathrm{a}}$ & $2,33 \pm 0,58$ \\
\hline CIP & $28,67 \pm 7,50^{\mathrm{b}}$ & $0,67 \pm 0,58^{\mathrm{a}, \mathrm{b}}$ & $6 \pm 0^{\mathrm{b}}$ \\
\hline FADANGA & $5 \pm 3,60^{c}$ & $0 \pm 0^{\mathrm{b}}$ & $2 \pm 0^{\mathrm{d}}$ \\
\hline POURPRE & $13,33 \pm 4,04^{\mathrm{b}, \mathrm{c}}$ & $0 \pm 0^{\mathrm{b}}$ & $5,67 \pm 0,58^{b}$ \\
\hline TIEBELE 2 & $6 \pm 4,58^{c}$ & $0 \pm 0^{\mathrm{b}}$ & $7,66 \pm 0,58$ \\
\hline$P(A N O V A)$ & 0,000712 & 0,008984 & 0,000001 \\
\hline
\end{tabular}

Les moyennes portant les mêmes lettres ne présentent pas de différences significatives selon les tests d'ANOVA et LSD de Fisher au seuil de $5 \%$.

\section{Discussion}

Les différents paramètres évalués au cours de cet essai ont révélé pour certains une différence significative et d'autres aucune différence.

Le taux de survie a varié d'une variété à une autre et d'un stade à un autre. Du stade plantule à la production, les variétés Irène, CIP et Covington ont respectivement enregistré les taux de survie les plus élevés. Ces variétés (Irène, CIP et Covington) ont obtenu des taux de survie similaires aux stades plantule et floraison. Par contre, au stade production la variété Covington s'est distinguée des deux autres avec le plus grand taux de survie. Les variétés Tiébélé 2; Pourpre; CIP 4 et Fadanga ont présenté des taux de survie largement inférieurs à celui de Irène. Ce résultat pourrait s'expliquer d'une part, par la vigueur des boutures et d'autre part, par l'adaptation aux aléas climatiques des différentes variétés mais aussi, par l'état de l'environnement dans lequel l'essai a été conduit. Des résultats similaires ont été obtenus par Ndekani (2014), au cours des travaux menés dans le sol de Mbanza-Ngungu (RDC Congo), où il a montré que le taux de levé était différent suivant les variétés en comparant les rendements de quatre (4) variétés d'arachide. Yves et al. (1997) soutiennent que les conditions pluviométriques qui ont régné pendant la reprise des boutures de patate douce ont influencé négativement la survie des variétés introduite.

La comparaison du poids des tiges aériennes, a révélé une différence significative entre les différentes variétés. Le poids de la biomasse aérienne d'Irène est largement supérieur à celui des six (6) variétés. Ce résultat pourraitêtre dû aux conditions de culture (climat et sol) qui étaient identiques pour toutes les variétés mises en essai. Ces conditions n'ont surement pas été favorables pour les six (6) variétés, surtout pour les variétés CIP 4 ; Fadanga et Tiébélé 2 qui ont obtenu les plus faibles poids en biomasse aérienne. 
Contrairement à Irène, qui s'adapte facilement à l'écologie auquel il est soumis, ces conditions de culture lui ont été très probablement favorables. En effet, la variation des conditions environnementales est l'un des paramètres qui agit sur les poids des tiges de patate douces. Djinet et al. (2015) confirment bien ce résultat en prouvant qu'il existe une différence significative entre la biomasse aérienne des dix variétés de patate douce qu'ils ont comparé dans deux conditions climatiques différentes.

L'incidence des ennemis de la patate douce a montré également des différences significatives entre la plupart des variétés. Globalement, toutes les variétés de patate douce ont été attaquées par les charançons. Cela serait imputable à la saison dans laquelle l'essai a été conduit notamment la saison sèche. Cette saison sèche, est favorable à l'accès des charançons aux racines tubéreuses de patate douce (Janssens, 2001). En effet, c'est pendant cette saison où il y'a manque d'eau dans le sol que les larves des charançons se multiplient et deviennent très actives dans le sol. Par ailleurs, les variétés Fadanga; Pourpre ; Tiébélé 2 et CIP 4 résistent très bien aux ravageurs vu qu'elles ont un pourcentage d'attaque inférieur à celui de CIP et Irène qui a une bonne résistance face à ces derniers. Seul Covington a eu un pourcentage d'attaque supérieur à celui d'Irène mais, cette valeur est relativement faible et on pourrait dire que celle-ci résiste également aux ravageurs. Aussi, la sévérité de l'attaque des virus est moins forte chez les variétés CIP 4 ; Covington et Fadanga que Irène. Ces dernières sont donc très résistantes face aux viroses comme c'est le cas chez Irène. Par contre les variétés CIP ; Pourpre et surtout Tiébélé 2 ne résistent pas aux viroses car elles ont été plus attaquées par les virus qu'Irène. Tout ceci pourrait être dû au milieu de culture et aussi aux caractères génétiques de chaque variété cultivée. Nos résultats sont en parfait accord avec ceux de Banganingwa (2011), dans l'étude menée sur le manioc à Bunia (RDC) et ses environs, qui affirme que la sensibilité à la mosaïque des variétés de manioc cultivées dépend à la fois des conditions du milieu et surtout de leur patrimoine génétique.

\section{Conclusion}

Cette étude a permis d'évaluer les caractères agronomiques de sept variétés de patate douce dans la région de Bouaké. Aux différents stades de croissance, les meilleurs taux de survie ont été enregistrés chez les variétés Irène, CIP et Covington. La variété Irene a produit plus de biomasse que les six autres variétés. Aussi, l'étude a montré que la variété Fadanga a été la moins attaquée par les charançons. En revanche seules les variétés CIP, Irène et Covington ont été affectés par les ravageurs. L'effet des virus a été plus sévère chez les variétés Tiébélé 2, CIP et Pourpre. Vu la performance des variétés Irène, CIP et Covington dans la région de Bouaké, elles pourraient 
très bien aussi s'adapter dans les autres zones agro écologiques du pays. Ainsi en termes de sélection, elles seraient bien indiquées.

\section{Remerciements}

Ce travail a été possible grâce au Centre National de Recherche Agronomique (CNRA), la fondation Helen Keller International (HKI) et la société Agri Ivoire Environnement (AIE).

\section{References:}

1. Allouko, J.R. (2011). Etude du prégrossissement d'Oreochromisniloticus en étang de rizipisciculture. Mémoire de fin d'étude de Diplôme d'Agronmie Approfondie, Institut National Félix Houphouët-Boigny, Yamoussoukro, Côte d'Ivoire, $50 \mathrm{pp}$.

2. Amani, N.V. (2017). Etude comparative de différentes techniques culturales (buttes, billons et labour à plat) sur le rendement de 5 génotypes de la patate douce dans les conditions pédoclimatiques de Kabare (cas du groupement de Bugorhe). Diplôme de Sciences Agronomiques Et Environnement, Université Evangélique En Afrique (Congo). Avril 2017. 36 pp.

3. Banganingwa, A.A. (2011). Etude sur l'incidence et sévérité de la mosaïque africaine du manioc à Bunia (RDC) et ses environs. Mémoire pour le grade d'ingénieur agronome, Université de Bunia RDC 48 pp.

4. Djinet, A.I., Nana, R., Tamini, Z. \& Badiel, B. (2015). Etude comparée des paramètres agromorphologiques de dix (10) variétés de patate douce (Ipomoea batatas (L.) Lam cultivées au champ dans deux (2) conditions climatiques au Tchad et au Burkina Faso. International Journal of Biological and Chemical Sciences, 9(3): 1243-1251. DOI: 10.4314/ijbcs.v9i3.9

5. FAOSTAT (2006). http://faostat.fao.org/site/567/default.aspx. (http:/www.ars-grin.gov/).

6. FAOSTAT (2018). http://faostat3.fao.org/download/Q/QC/E, consulté le 23 mai 2018.

7. Hironori, M., Ogasawara, F., Sato, K., Higo, H. and Minobe, Y. 2007. Isolation of a regulatory gene of anthocyanin biosynthesis in tuberous roots of purple-fleshed sweet potato. Plant Physiology, 143: 12521268. DOI: https://doi.org/10.1104/pp.106.094425

8. Janssens, M.J.J. (2001). Sweet potatoes (Ipomoea batatas L) Lam: In Crop Production in the tropical Romain H Raemaekersed. pp 205-220.

9. Karyeija, R.F., Gibson, R.W.J. \& Valkonen, P.T. (1998). The significance of sweet potato feathery mottle virus in subsistence sweet potato production in Africa. Plant Disease, 82 : 4-15. 
10. Ndekani, M.C. (2014). Etude comparative sur les rendements des 4 variétés d'arachide dans le sol de Mbanza-Ngungu. Mémoire de fin de cycle, faculté des sciences agronomiques, Université Kongo, Kisantu, République Démocratique Congo, pp 38.

11. Yesso, P., Kone, D. \& Meyer, C. (1991). Reprise post-partum et cyclicité des vaches trypanotolérantes en fonction de la variation saisonnière en région centre Côte d'Ivoire In : IAEA sur l'Amélioration de la Productivité du Bétail Indigène Africain en Utilisant les Méthodes Radio-immunologiques et Apparentées. Réunion de Coordination FAO. 3, Bouaké, IDESSA, pp 22.

12. Yves, J., Dennis, A., Shannon, F., Brockman, E. \& Julène, M. (1997). Essai d'adaptation de variétés de patate douce (Ipomea batatas) aux conditions de culture du Nord-Ouest d'Haïti. Productive land use systems project Haïti. pp 52. 